\title{
Adult attention-deficit hyperactivity disorder and obesity: epidemiological study
}

Samuele Cortese, Stephen V. Faraone, Silvia Bernardi, Shuai Wang and Carlos Blanco

\section{Background}

A significant association between attention-deficit hyperactivity disorder (ADHD) and obesity has been reported. This study addresses unexplored aspects of this relationship.

\section{Aims}

To evaluate the association between adult obesity and: (a) persistent, remitted or lifetime ADHD; (b) number of childhood ADHD symptoms, controlling for socioeconomic status and mood, anxiety and substance use disorders.

\section{Method}

Face-to-face psychiatric interviews in 34653 US adults from the National Epidemiologic Study on Alcohol and Related Conditions. Obesity was defined as a body mass index $\geqslant 30$.

\section{Results}

Persistent, lifetime or remitted ADHD were not associated with obesity after controlling for confounders. The number of childhood ADHD symptoms was significantly associated with adult obesity, even after adjustment, in women.

\section{Conclusions}

Childhood ADHD symptoms are associated with obesity in women even after comorbid psychiatric disorders are accounted for. This provides a rationale for longitudinal studies assessing the impact of the treatment of childhood ADHD symptoms on obesity in women.

\section{Declaration of interest}

S.C. has served as scientific consultant for Shire Pharmaceuticals (2009-2010). In the past year, S.V.F. received consulting income and/or research support from Shire, Otsuka and Alcobra, and research support from the National Institutes of Health (NIH). He is also on the Clinical Advisory Board for Akili Interactive Labs. In previous years, he received consulting fees or was on advisory boards or participated in continuing medical education programmes sponsored by: Shire, McNeil, Janssen, Novartis, Pfizer, and Eli Lilly.
Attention-deficit hyperactivity disorder (ADHD) is one of the most frequent childhood-onset psychiatric conditions, with an estimated worldwide prevalence exceeding $5 \%$ in school-age children. ${ }^{1}$ Impairing symptoms of ADHD persist into adulthood in approximately $65 \%$ of childhood-onset cases. ${ }^{2}$ Attention-deficit hyperactivity disorder imposes an enormous burden on society in terms of psychological dysfunction, adverse vocational outcomes, stress on families and societal financial costs. ${ }^{3}$ Whereas the comorbidity between ADHD and psychiatric disorders has been extensively investigated, ${ }^{3}$ the association with general medical conditions has received much less attention. Among medical disorders, there is increasing, but in part mixed evidence, that points to a significant association between obesity and ADHD in children. ${ }^{4}$ Although literature in adults is limited, initial evidence from population samples suggests a persistence of a significant association in adulthood as well. ${ }^{4}$ Defined as a body mass index $(\mathrm{BMI}) \geqslant 30,{ }^{5}$ obesity is a chronic condition currently affecting one adult in three in the USA. ${ }^{6}$ It is considered one of the major causes of morbidity (including cardiovascular risk and diabetes) and mortality in adults. ${ }^{6}$ Given the significant impairment associated with both ADHD and obesity, adults with both conditions represent a portion of the population particularly vulnerable from a medical and psychosocial standpoint. Interestingly, preliminary data suggest that the treatment of ADHD may also contribute to significant weight reduction in adults with ADHD and obesity. ${ }^{7}$ However, before implementing screening and intervention programmes aimed at preventing or reducing obesity in adults through ADHD treatment, it is necessary to extend current preliminary literature, addressing unexplored important aspects that may inform such programmes.

A question that remains unanswered is whether the association between ADHD and obesity in adults holds after controlling for possible confounders. In particular, it is unclear whether ADHD is associated with obesity after taking into account the effect of comorbid mental disorders that have been shown to have an impact on weight, i.e. mood, anxiety and substance use disorders. ${ }^{8}$ Determining whether adult ADHD per se is associated with obesity or whether the link is mediated by other factors, including comorbid psychiatric disorders, is crucial before implementing possible interventions strategies for the management of obesity based on the specific treatment of ADHD.

Moreover, although there is evidence of a relationship between persistent ADHD and obesity in adulthood, ${ }^{4}$ it is not known whether ADHD in remission is associated with obesity as well. This would be highly relevant from a clinical and public health perspective, setting the groundwork for prospective studies assessing the effects of ADHD treatment in youth as a way to prevent obesity in adulthood. Additionally, focusing only on a categorical definition of ADHD may limit our ability to detect a significant association with obesity. Whereas both impulsive and inattentive symptoms of ADHD have been hypothesised to lead to dysregulated eating behaviours that contribute to weight gain and obesity, the role of hyperactivity is unclear. ${ }^{4}$ Considering the three symptom dimensions of ADHD separately (i.e. inattentive, impulsive and hyperactive symptoms) may allow us to better understand specific behavioural and neurobiological pathways underlying the possible association between ADHD symptoms and obesity. Taking into account the three ADHD symptom dimensions separately may also be more informative for specific preventive and intervention strategies addressing one or more of these dimensions. So far, only one study ${ }^{9}$ has addressed the relationship between ADHD symptoms (lumping together hyperactive and impulsive symptoms) in childhood and obesity in early adulthood, but it has not been explored whether 
childhood ADHD symptoms are associated with obesity in mid-adulthood and later on, when the prevalence of obesity is the highest. ${ }^{6}$ Finally, there is some evidence that the association between ADHD and obesity depends on gender, with one study in children and adolescents reporting a significant association between ADHD symptoms and obesity only in adolescents females, ${ }^{10}$ and two other studies ${ }^{11,12}$ pointing to higher odds ratios for the association between ADHD and obesity in unmedicated female adolescents than in male adolescents. Therefore, there is a need to better understand possible gender differences in the association between ADHD and adult obesity.

To address these unexplored issues, we draw on data from the National Epidemiologic Survey on Alcohol and Related Conditions (NESARC), ${ }^{13}$ a large representative sample of the US adult population. The NESARC assessed, by means of face-to-face interviews, a broad range of Axis I and II disorders as well as several general medical conditions, including obesity. The aims of the present study were: (a) to assess the relationship between persistent, lifetime or remitted ADHD and obesity in adulthood, controlling for relevant sociodemographic variables as well as for mood, anxiety and substance use disorders; and (b) to evaluate the relationship of obesity in adulthood to each of ADHD's symptom dimensions in childhood, controlling for the aforementioned possible confounders. Analyses were performed in the entire sample (i.e. men plus women), as well as in men and women separately. Based on prior literature, ${ }^{4}$ we hypothesised: (a) a significant positive association between obesity in adulthood and persistent, as well as lifetime and remitted ADHD, even after controlling for relevant sociodemographic characteristics and comorbid mental disorders, in women but not in men; and (b) a significant relationship of obesity in adulthood to impulsive and inattentive but not hyperactive symptoms of ADHD in childhood, that holds after controlling for relevant sociodemographic and psychiatric confounders, in women but not in men.

\section{Method}

\section{Sample and procedures}

The NESARC ${ }^{13}$ target population at Wave 1 (2001-2002) was the civilian non-institutionalised population 18 years and older residing in households and group quarters (such as college halls of residents, barracks, residential treatment centres) in the USA, including Alaska and Hawaii. One sample individual from each household or group quarters' unit was randomly selected for interview. Black and Hispanic people, and adults 18-24 years were oversampled, with data adjusted for oversampling and household and individual-level non-response. Black and Hispanic people and young adults were oversampled because these subgroups have been underrepresented in previous comorbidity surveys in the USA. ${ }^{14}$ Interviews were conducted with 43093 participants by experienced lay interviewers with extensive training and supervision. All procedures, including informed consent, received full human subjects review and approval from the US Census Bureau and the US Office of Management and Budget. The Wave 2 interview was conducted approximately 3 years later (2004-2005, mean interval 36.6 months $($ s.e.=2.6)). Excluding ineligible respondents (for example deceased), the Wave 2 response rate was $86.7 \%$, reflecting 34653 completed interviews. ${ }^{15}$ Participants were $\geqslant 20$ years old (individuals aged 90 or older were coded as 90 for privacy purposes). Wave 2 NESARC weights include a component that adjusts for nonresponse, demographic factors and psychiatric diagnoses to ensure that the Wave 2 sample approximated the target population, that is, the original sample minus attrition between the two waves. As reported elsewhere, ${ }^{15}$ adjustment for non-response was successful, as the Wave 2 respondents and the original target population did not differ in age, ethnicity, gender, socioeconomic status or the presence of any substance, mood, anxiety or personality disorder. The analyses of the present study refer to participants in Wave 2.

\section{Measures}

\section{Sociodemographic and socioeconomic variables}

Sociodemographic measures presented in this study include age, gender, ethnicity, nativity, education and individual income.

\section{DSM-IV diagnoses}

The diagnostic interview Alcohol Use Disorder and Associated Disabilities Interview Schedule-DSM-IV Version (AUDADIS-IV) Wave 2 version ${ }^{16}$ was used to generate DSM-IV diagnoses. ${ }^{17}$ The AUDADIS-IV is a valid and reliable fully structured diagnostic interview designed for use by professional interviewers who are not clinicians.

ADHD. Respondents were asked about symptoms of DSM-IV ADHD in Wave 2 only. Twenty symptom items operationalised the $18 \mathrm{ADHD}$ items of DSM-IV criterion A. For the diagnosis of ADHD, six or more inattention and/or impulsive-hyperactive symptoms had to be present for at least 6 months (criterion A), be associated with impairment in two or more settings (criterion C) and interfere significantly with social, school or work functioning (criterion D). To be consistent with empirical data showing validity of late-onset diagnosis and with the recommendation of the DSM-5 committee, ${ }^{18}$ symptoms had to be present before the age of 12 , rather than before age 7 as currently required by DSM-IV(-TR). ${ }^{19}$ Persistent ADHD was defined by the current presence of the aforementioned criteria at Wave 2. Remitted ADHD was defined as at least 2 months without symptoms at Wave 2. Lifetime ADHD was defined as persistent plus remitted ADHD. Test-retest reliability for ADHD was good $(\kappa=0.71) .{ }^{20}$ Internal consistency reliability of the ADHD symptom items (Cronbach's $\alpha=0.89$ ) was excellent. ${ }^{20}$

Other DSM-IV diagnoses. Mood disorders assessed by the AUDADIS-IV included major depressive disorder, dysthymia, and bipolar I and II disorder. Anxiety disorders included DSM-IV panic disorder, social anxiety disorder, specific phobia, generalised anxiety disorder and post-traumatic stress disorder (PTSD). Personality disorders were assessed on a lifetime basis at Wave 1 and included avoidant, dependent, obsessivecompulsive, paranoid, schizoid, histrionic and antisocial personality disorders. Borderline, schizotypal and narcissistic personality disorders were measured at Wave 2. Test-retest reliabilities for AUDADIS-IV mood, anxiety, impulsive and personality disorders in the general population and clinical settings were fair to good $(\kappa=0.40-0.77) .{ }^{20}$ Convergent validity was good to excellent for all affective, anxiety and personality diagnoses, and selected diagnoses showed good agreement $(\kappa=0.64-0.68)$ with psychiatrist reappraisals. ${ }^{20}$ The AUDADIS-IV also has good to excellent $(\kappa=0.70-0.91)$ test-retest reliability for substance use disorder diagnoses. $^{20}$

\section{Obesity}

Current (i.e. at Wave 2) weight (in pounds) and height (in inches) were self-reported at Wave 2. Body mass index was calculated as follows: ${ }^{5}$ (weight (pounds)/height (inches) $\left.{ }^{2}\right) \times 703$. Obesity was defined as $\mathrm{BMI} \geqslant 30$, consistent with World Health Organization (WHO) criteria. $^{21}$ 


\section{Statistical analysis}

Weighted percentages and means were computed to derive sociodemographic and clinical characteristics, including DSM-IV diagnoses, as well as current weight, height, BMI and obesity rates of respondents with and without a diagnosis of ADHD (persistent, remitted or lifetime). Data were weighted to adjust for the sample design, for the effects of non-response and to correct for survey undercoverage error (adjusting by age, gender and ethnicity). To assess the first aim of the study, logistic regression yielded odds ratios (ORs) and 95\% CIs indicating measures of association between ADHD diagnosis (persistent, remitted or lifetime) and obesity (as well as height, weight and BMI). To control for sociodemographic and psychiatric variables, two sets of logistic regressions, yielding adjusted ORs and 95\% CIs, were conducted. The first included current obesity status as the outcome variable and persistent ADHD, as well as 12-month mood disorders, anxiety disorders, substance use disorders, ethnicity and individual income as predictors. All these variables have been found to be associated with ADHD in a previous report on this sample ${ }^{22}$ and with obesity in a prior national survey. ${ }^{8}$ With regard to substance use disorders, we used 'nicotine dependence' and 'any substance use disorders, other than nicotine dependence' as independent variables, to specifically assess the role of nicotine dependence. The second logistic regression model was similar to the previous one, but included lifetime instead of persistent $\mathrm{ADHD}$, and lifetime rather than 12-month mood disorders, anxiety disorders and substance use disorders (again, considering 'nicotine dependence' and 'any substance use disorders, other than nicotine dependence').

To evaluate the second aim of the study, a logistic regression model was developed including current obesity as the outcome variable and number of inattentive, hyperactive and impulsive symptoms (separately) before age 18, along with the other variables included in the aforementioned second model, as predictors. All regression models, and the prevalence of anthropometric measures, are presented in the overall sample (i.e. men plus women) as well as stratified by gender. We consider significant ORs those whose confidence intervals does not include 1. Standard errors and 95\% CIs for all analyses were estimated by using SUDAAN (version 9.0) on Windows, to adjust for the design effects of the NESARC.

\section{Results}

\section{Sociodemographic, socioeconomic and clinical characteristics}

Sociodemographic and socioeconomic characteristics of the sample are shown in Table 1 and lifetime mental disorders in online Table DS1. There were 14564 males in the full sample (47.92\%, weighted percentage). The prevalence of persistent, remitted and lifetime ADHD were 1.02, 0.91 and 1.93\% respectively. Details about the clinical characteristics of the adults with ADHD in this sample have been previously described. ${ }^{22}$

\section{Obesity rates in adults with persistent, remitted and lifetime ADHD}

Considering the entire sample (men plus women), obesity rates, as well as BMI (Table 2), were significantly higher in adults with persistent ADHD than in those without ADHD (obesity: $\mathrm{OR}=1.44,95 \%$ CI 1.06-1.95; BMI: $P=0.015)$. Body mass index $(P=0.032)$, but not obesity rates, was also significantly higher in adults with lifetime ADHD v. non-ADHD. Adults with ADHD (persistent, remitted or lifetime) had significantly higher weight than individuals without $\mathrm{ADHD}(P=0.005, \quad P=0.001$ and $P=0.001$ respectively). However, results were different when stratifying by gender. In men, BMI and obesity rates did not differ significantly across groups (Table 3 ). On the contrary, women with lifetime ADHD presented with significantly higher BMI $(P=0.019)$ and obesity rates $(\mathrm{OR}=1.41,95 \% \mathrm{CI} 1.05-1.89)$ than women without ADHD (Table 4). In addition, women with persistent ADHD had significantly higher obesity rates $(\mathrm{OR}=1.48,95 \%$ CI 1.01-2.17) than women without ADHD (Table 4). Moreover, weight was significantly higher in men with persistent ADHD compared with men without ADHD $(P=0.045$, Table 3$)$ and in women with lifetime ADHD in relation to women without ADHD $(P=0.019$, Table 4$)$.

In the entire sample, after controlling for sociodemographic and 12-month DSM-IV diagnoses (Table 5), persistent ADHD was no longer significantly associated with current obesity $(P=0.075)$. The lack of significant association between persistent ADHD and current obesity after controlling for the aforementioned factors was confirmed when limiting the analyses to men or women, separately (Tables 6 and 7 respectively). After entering each of the confounding variables in the model separately, persistent ADHD was still significantly associated with obesity controlling for substance use disorders or ethnicity or income, but was not significantly associated with obesity controlling for mood or anxiety disorders (data available from the authors on request). There was no significant association between lifetime ADHD and obesity after controlling for other lifetime DSM-IV diagnoses, in the entire sample as well as when considering the two genders separately (online Tables DS2-4).

\section{Relationship of ADHD symptom dimensions in childhood to obesity in adulthood}

In the entire sample, after controlling for lifetime DSM-IV diagnoses and sociodemographic characteristics, the number of impulsive $(P=0.001)$ and inattentive $(P=0.036)$, but not of hyperactive symptoms before age 18 , was significantly associated with current obesity in adulthood (Table 8). However, a different pattern emerged when considering the two genders separately. In men, the number of impulsive, inattentive or hyperactive symptoms before 18 years was not significantly associated with current obesity rates (the association with the number of impulsive symptoms approached significance: $P=0.06$ ) (Table 9). By contrast, in women, the number of each of the three symptom dimensions before 18 years was significantly associated with current obesity (Table 10).

\section{Discussion}

This is the largest study to examine the relationship between $\mathrm{ADHD}$ and obesity. It is also the first one to relate ADHD as a categorical construct and each ADHD symptom dimension in childhood to obesity in adulthood controlling for an extensive set of confounding factors, including sociodemographic variables and DSM diagnoses (mood disorders, anxiety disorders, and substance use disorders) and to perform these analyses stratified by gender. Our study provides insights into unexplored issues that may inform further screening and treatment programmes aimed at decreasing obesity by means of ADHD treatment.

The results of this study did not support our hypothesis of a significant association, in women, between ADHD and obesity after controlling for a broad range of covariates. Whereas, in the unadjusted model, persistent ADHD was significantly associated with obesity in adulthood in the entire sample as well as in women, the relationship did not hold after controlling for 


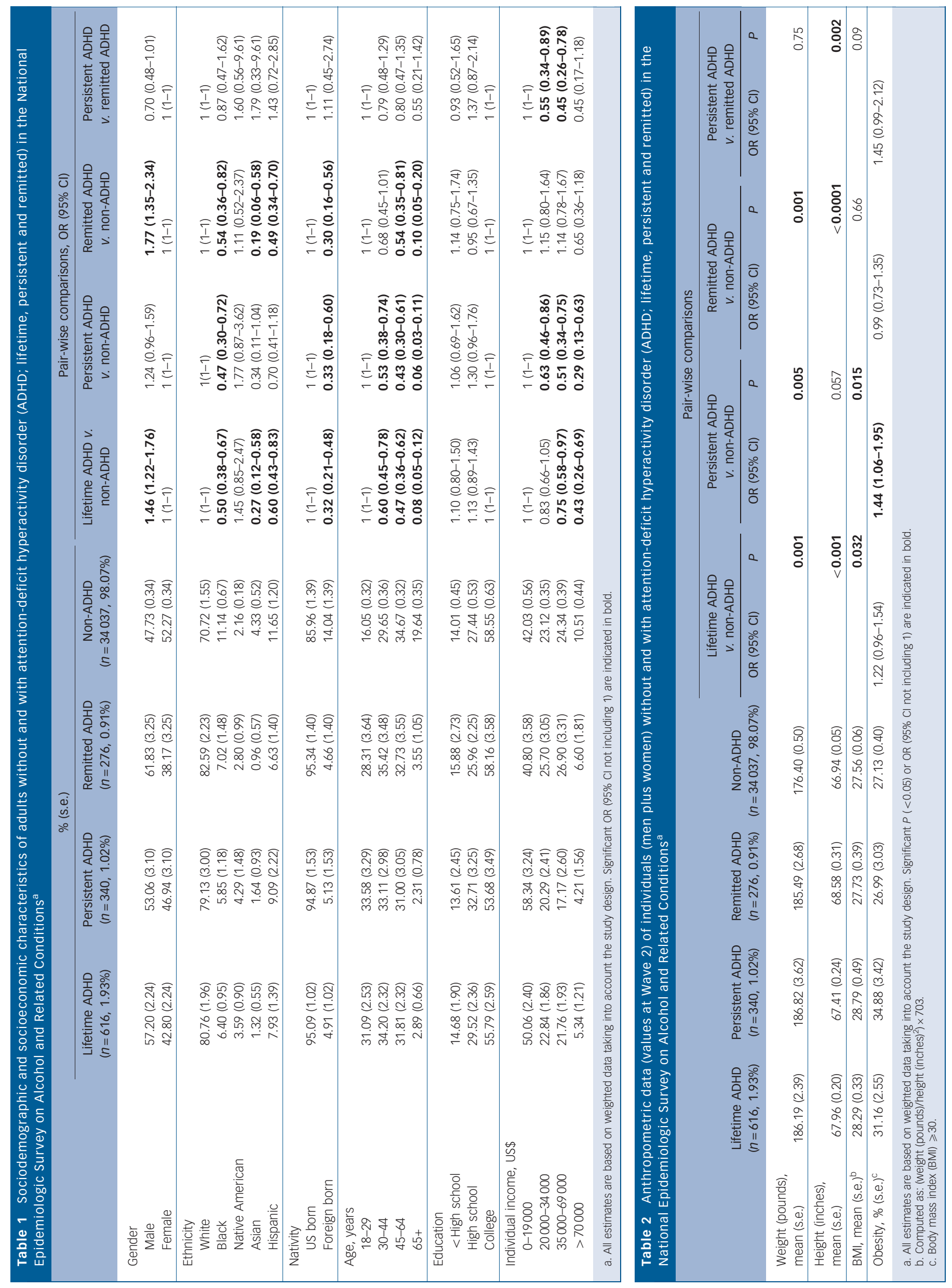



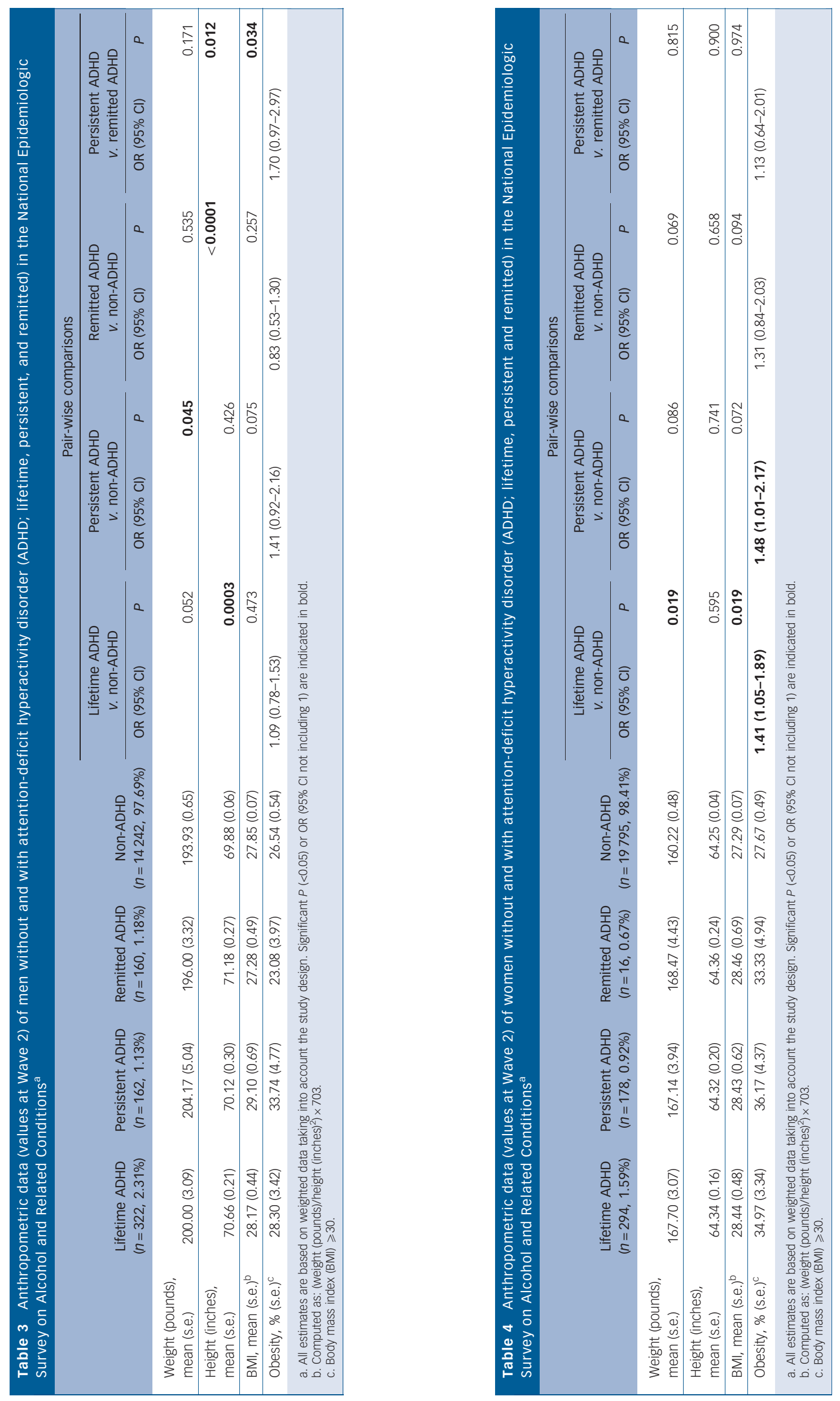


\begin{tabular}{|c|c|c|c|c|c|}
\hline Predictors (independent variables) & $b$ & s.e. & Adjusted OR & $95 \% \mathrm{Cl}$ & $P$ (2-tailed) \\
\hline Persistent attention-deficit hyperactivity disorder & 0.27 & 0.15 & 1.31 & $0.97-1.78$ & 0.075 \\
\hline Mood disorder, 12-month & 0.27 & 0.05 & 1.30 & $1.18-1.44$ & $<0.0001$ \\
\hline Anxiety disorder, 12-month & 0.24 & 0.04 & 1.27 & $1.16-1.39$ & $<0.0001$ \\
\hline Any substance use disorder other than nicotine dependence, 12-month & -0.20 & 0.05 & 0.82 & $0.74-0.91$ & 0.0004 \\
\hline Nicotine dependence, 12-month & -0.11 & 0.05 & 0.89 & $0.81-0.99$ & 0.0302 \\
\hline \multicolumn{6}{|l|}{ Ethnicity } \\
\hline White & 0.00 & 0.00 & 1.00 & $1.00-1.00$ & - \\
\hline Black & 0.59 & 0.04 & 1.80 & $1.66-1.94$ & $<0.0001$ \\
\hline Native American & 0.50 & 0.11 & 1.64 & $1.33-2.03$ & $<0.0001$ \\
\hline Asian & -1.16 & 0.14 & 0.31 & $0.24-0.42$ & $<0.0001$ \\
\hline Hispanic & 0.21 & 0.05 & 1.24 & $1.12-1.37$ & 0.0001 \\
\hline \multicolumn{6}{|l|}{ Individual income, US\$ } \\
\hline 0-19000 & 0.00 & 0.00 & 1.00 & $1.00-1.00$ & - \\
\hline $20000-34000$ & 0.00 & 0.04 & 1.00 & $0.92-1.09$ & 0.982 \\
\hline $35000-69000$ & 0.02 & 0.04 & 1.02 & $0.95-1.09$ & 0.655 \\
\hline$>70000$ & 0.14 & 0.06 & 0.87 & $0.78-0.98$ & 0.0201 \\
\hline
\end{tabular}

\begin{tabular}{|c|c|c|c|c|c|}
\hline Predictors (independent variables) & $b$ & s.e. & Adjusted OR & $95 \% \mathrm{Cl}$ & $P$ (2-tailed) \\
\hline Persistent attention-deficit hyperactivity disorder & 0.29 & 0.21 & 1.33 & $0.88-2.03$ & 0.176 \\
\hline Mood disorder, 12-month & 0.11 & 0.10 & 1.11 & $0.92-1.35$ & 0.277 \\
\hline Anxiety disorder, 12-month & 0.26 & 0.08 & 1.30 & $1.11-1.52$ & 0.001 \\
\hline Any substance use disorder other than nicotine dependence, 12-month & -0.10 & 0.06 & 0.91 & $0.80-1.03$ & 0.132 \\
\hline Nicotine dependence, 12-month & -0.10 & 0.07 & 0.91 & $0.79-1.05$ & 0.188 \\
\hline \multicolumn{6}{|l|}{ Ethnicity } \\
\hline White & 0.00 & 0.00 & 1.00 & $1.00-1.00$ & - \\
\hline Black & 0.27 & 0.07 & 1.31 & $1.15-1.50$ & 0.0001 \\
\hline Native American & 0.59 & 0.16 & 1.80 & $1.30-2.48$ & 0.0005 \\
\hline Asian & -0.98 & 0.20 & 0.38 & $0.25-0.56$ & $<0.0001$ \\
\hline Hispanic & 0.14 & 0.06 & 1.15 & $1.02-1.30$ & 0.0237 \\
\hline \multicolumn{6}{|l|}{ Individual income, US\$ } \\
\hline 0-19000 & 0.00 & 0.00 & 1.00 & $1.00-1.00$ & - \\
\hline $20000-34000$ & 0.09 & 0.06 & 1.10 & $0.97-1.24$ & 0.1449 \\
\hline $35000-69000$ & 0.19 & 0.05 & 1.21 & $1.09-1.35$ & 0.0005 \\
\hline$>70000$ & 0.02 & 0.08 & 1.02 & $0.87-1.20$ & 0.8236 \\
\hline
\end{tabular}

sociodemographic characteristics and DSM-IV diagnoses associated with both ADHD and obesity. Lifetime ADHD was associated with current obesity only in women and only in the unadjusted model. However, when considering ADHD symptom dimensions, the number of lifetime inattentive, impulsive and hyperactive symptoms (separately) before age 18 was significantly associated with obesity in adulthood in women, even after controlling for confounding variables, as predicted by our second hypothesis. In men, no symptom dimension was significantly associated with current obesity in adulthood.

\section{Relationship between ADHD as a categorical diagnosis and obesity}

The association, in the unadjusted model, between persistent $\mathrm{ADHD}$ and obesity in the entire sample is in line with prior epidemiological studies in adults and in children, summarised in Cortese \& Vincenzi ${ }^{4}$ (with the exception of one epidemiological study in early adolescents ${ }^{23}$ ), including mixed samples of males and females. Our study, as in the one by Pagoto et al, ${ }^{24}$ used face-to-face interviews to generate a formal diagnosis of ADHD. Other epidemiological studies relied on self-ratings in adult samples, parent/teacher rating scales in children, interviews assessing ADHD symptoms without a formal diagnosis of ADHD or, in childhood samples, on a single question such as 'Has your doctor ever told you that your child has ADHD?" ${ }^{4}$ Both selfratings and the use of a single question have lower reliability compared with face-to-face interviews. ${ }^{25}$ As in Pagoto et al, ${ }^{24}$ we found that remitted ADHD was not significantly associated with current obesity, whereas persistent ADHD was. This suggests that the presence of a categorical diagnosis of ADHD in the past would not be sufficient, per se, to account for the increased likelihood of current obesity in adulthood. This finding concurs with the report by Barkley et al who, in their longitudinal cohort, found significantly higher BMI in individuals with persistent, but not remitted, ADHD, compared with individuals without ADHD (obesity rates were not reported in that study). ${ }^{26}$ We note that the prevalence of obesity in our non-ADHD sample $(27.1 \%$, 95\% CI 26.35-27.91) was close to the overall self-reported obesity rate in the USA $(26.7 \%, 95 \%$ CI $26.4-27.0)$ in $2009 .{ }^{27}$ Therefore, it 


\begin{tabular}{|c|c|c|c|c|c|}
\hline Predictors (independent variables) & $b$ & s.e. & Adjusted OR & $95 \% \mathrm{Cl}$ & $P$ (2-tailed) \\
\hline Persistent attention-deficit hyperactivity disorder & 0.26 & 0.20 & 1.29 & $0.87-1.92$ & 0.1950 \\
\hline Mood disorder, 12-month & 0.37 & 0.06 & 1.44 & $1.28-1.62$ & $<0.0001$ \\
\hline Anxiety disorder, 12-month & 0.23 & 0.05 & 1.26 & $1.14-1.40$ & $<0.0001$ \\
\hline Any substance use disorder other than nicotine dependence, 12-month & -0.42 & 0.09 & 0.66 & $0.55-0.79$ & $<0.0001$ \\
\hline Nicotine dependence, 12-month & -0.12 & 0.07 & 0.88 & $0.77-1.01$ & 0.0667 \\
\hline \multicolumn{6}{|l|}{ Ethnicity } \\
\hline White & 0.00 & 0.00 & 1.00 & $1.00-1.00$ & - \\
\hline Black & 0.84 & 0.04 & 2.32 & $2.12-2.54$ & $<0.0001$ \\
\hline Native American & 0.42 & 0.14 & 1.52 & $1.16-2.01$ & 0.0033 \\
\hline Asian & -1.36 & 0.18 & 0.26 & $0.18-0.37$ & $<0.0001$ \\
\hline Hispanic & 0.28 & 0.07 & 1.32 & $1.15-1.52$ & 0.0002 \\
\hline \multicolumn{6}{|l|}{ Individual income, US\$ } \\
\hline 0-19000 & 0.00 & 0.00 & 1.00 & $1.00-1.00$ & - \\
\hline $20000-34000$ & -0.05 & 0.06 & 0.95 & $0.85-1.06$ & 0.3827 \\
\hline $35000-69000$ & -0.18 & 0.05 & 0.84 & $0.75-0.93$ & 0.0017 \\
\hline$>70000$ & -0.50 & 0.10 & 0.61 & $0.50-0.74$ & $<0.0001$ \\
\hline
\end{tabular}

\begin{tabular}{|c|c|c|c|c|c|}
\hline Predictors (independent variables) & $b$ & s.e. & Adjusted OR & $95 \% \mathrm{Cl}$ & $P$ (2-sided) \\
\hline Number of inattentive symptoms & 0.02 & 0.01 & 1.02 & $1.00-1.05$ & 0.036 \\
\hline Number of impulsive symptoms & 0.06 & 0.02 & 1.06 & $1.02-1.10$ & 0.001 \\
\hline Number of hyperactive symptoms & -0.02 & 0.01 & 0.98 & $0.95-1.00$ & 0.092 \\
\hline Any mood disorders, lifetime & 0.26 & 0.03 & 1.30 & $1.21-1.39$ & $<0.0001$ \\
\hline Any anxiety disorders, lifetime & 0.20 & 0.04 & 1.22 & $1.13-1.31$ & $<0.0001$ \\
\hline Any substance use disorders (other than nicotine dependence), lifetime & -0.08 & 0.04 & 0.92 & $0.86-0.99$ & 0.028 \\
\hline Nicotine dependence, lifetime & -0.06 & 0.04 & 0.94 & $0.87-1.02$ & 0.121 \\
\hline \multicolumn{6}{|l|}{ Ethnicity } \\
\hline White & 0.00 & 0.00 & 1.00 & $1.00-1.00$ & \\
\hline Black & 0.60 & 0.04 & 1.82 & $1.68-1.96$ & $<0.0001$ \\
\hline Native American & 0.48 & 0.11 & 1.61 & $1.30-1.99$ & $<0.0001$ \\
\hline Asian & -1.17 & 0.15 & 0.31 & $0.23-0.42$ & $<0.0001$ \\
\hline Hispanic & 0.22 & 0.05 & 1.25 & $1.13-1.38$ & $<0.0001$ \\
\hline \multicolumn{6}{|l|}{ Individual income, US\$ } \\
\hline 0-19000 & 0.00 & 0.00 & 1.00 & $1.00-1.00$ & \\
\hline $20000-34000$ & 0.02 & 0.04 & 1.02 & $0.94-1.11$ & 0.646 \\
\hline $35000-69000$ & 0.03 & 0.04 & 1.04 & $0.96-1.12$ & 0.358 \\
\hline$>70000$ & -0.10 & 0.06 & 0.90 & $0.80-1.01$ & 0.071 \\
\hline
\end{tabular}

is unlikely that the significantly higher obesity rates in individuals with ADHD in our sample were the result of unusually low obesity rate in individuals without ADHD.

Although we confirmed the significant association between persistent ADHD and obesity at the unadjusted level, as already reported in prior smaller epidemiological studies (summarised in Cortese \& Vincenzi ${ }^{4}$ ), this association did not hold after controlling for a broad range of variables including sociodemographic variables and a wide range of DSM-IV diagnoses (mood disorders, anxiety disorders and substance use disorders) in the entire sample as well as when stratifying by gender. These results did not significantly change when we considered lifetime ADHD and lifetime mental disorders instead of 12-month diagnoses. Prior studies controlled for comorbid major depressive disorder ${ }^{24}$ or for symptoms of depression/anxiety (without a formal diagnosis). ${ }^{28}$ However, to our knowledge, our study is the first to include, as covariates, the broader range of mood disorders as well as anxiety disorders and substance use disorders diagnosed with face-to-face interviews.

Our results suggest that, although adults with ADHD may be at risk of obesity, comorbid mood and depressive disorders might be more directly linked to obesity than ADHD per se. Therefore, if further longitudinal studies confirm a causal relationship between comorbid mood and anxiety disorders and obesity in adults with $\mathrm{ADHD}$, specific screening and intervention programmes for the management of obesity in adults with ADHD should take into account comorbid mood and anxiety disorders.

\section{Relationship between ADHD symptom dimensions and obesity}

In the entire sample, hyperactive symptoms before age 18 were not significantly associated with obesity after controlling for relevant covariates; on the other hand, the number of inattentive or 


\begin{tabular}{|c|c|c|c|c|c|}
\hline Predictors (independent variables) & $b$ & s.e. & Adjusted OR & $95 \% \mathrm{Cl}$ & $P$ (2-sided) \\
\hline Number of inattentive symptoms & 0.02 & 0.02 & 1.02 & $0.99-1.05$ & 0.260 \\
\hline Number of impulsive symptoms & 0.05 & 0.03 & 1.05 & $1.00-1.11$ & 0.061 \\
\hline Number of hyperactive symptoms & -0.02 & 0.02 & 0.98 & $0.95-1.02$ & 0.378 \\
\hline Any mood disorders, lifetime & 0.21 & 0.06 & 1.23 & $1.08-1.40$ & 0.001 \\
\hline Any anxiety disorders, lifetime & 0.12 & 0.06 & 1.12 & $1.00-1.26$ & 0.052 \\
\hline Any substance use disorders (other than nicotine dependence), lifetime & 0.00 & 0.05 & 1.00 & $0.91-1.10$ & 0.981 \\
\hline Nicotine dependence, lifetime & -0.07 & 0.06 & 0.94 & $0.83-1.05$ & 0.258 \\
\hline \multicolumn{6}{|l|}{ Ethnicity } \\
\hline White & 0.00 & 0.00 & 1.00 & $1.00-1.00$ & \\
\hline Black & 0.29 & 0.07 & 1.34 & $1.17-1.53$ & 0.0001 \\
\hline Native American & 0.56 & 0.16 & 1.75 & $1.26-2.41$ & 0.001 \\
\hline Asian & -0.98 & 0.20 & 0.37 & $0.25-0.56$ & $<0.0001$ \\
\hline Hispanic & 0.15 & 0.06 & 1.16 & $1.03-1.32$ & 0.019 \\
\hline \multicolumn{6}{|l|}{ Individual income, US\$ } \\
\hline 0-19000 & 0.00 & 0.00 & 1.00 & $1.00-1.00$ & \\
\hline $20000-34000$ & 0.12 & 0.06 & 1.13 & $0.99-1.28$ & 0.065 \\
\hline $35000-69000$ & 0.21 & 0.05 & 1.24 & $1.11-1.38$ & 0.0001 \\
\hline$>70000$ & 0.05 & 0.08 & 1.05 & $0.89-1.23$ & 0.548 \\
\hline
\end{tabular}

\begin{tabular}{|c|c|c|c|c|c|}
\hline Predictors (independent variables) & $b$ & s.e. & Adjusted OR & $95 \% \mathrm{Cl}$ & $P$ (2-sided) \\
\hline Number of inattentive symptoms & 0.03 & 0.01 & 1.03 & $1.00-1.05$ & 0.0372 \\
\hline Number of impulsive symptoms & 0.08 & 0.02 & 1.09 & $1.03-1.14$ & 0.0014 \\
\hline Number of hyperactive symptoms & -0.04 & 0.02 & 0.96 & $0.92-1.00$ & 0.0454 \\
\hline Any mood disorders, lifetime & 0.33 & 0.05 & 1.39 & $1.27-1.52$ & $<0.0001$ \\
\hline Any anxiety disorders, lifetime & 0.27 & 0.05 & 1.31 & $1.19-1.43$ & $<0.0001$ \\
\hline Any substance use disorders (other than nicotine dependence), lifetime & -0.20 & 0.05 & 0.81 & $0.73-0.90$ & 0.0002 \\
\hline Nicotine dependence, lifetime & -0.06 & 0.05 & 0.94 & $0.85-1.05$ & 0.242 \\
\hline \multicolumn{6}{|l|}{ Ethnicity } \\
\hline White & 0.00 & 0.00 & 1.00 & $1.00-1.00$ & - \\
\hline Black & 0.84 & 0.04 & 2.33 & $2.13-2.54$ & 0.0001 \\
\hline Native American & 0.41 & 0.14 & 1.51 & $1.13-2.00$ & 0.0052 \\
\hline Asian & -1.36 & 0.18 & 0.26 & $0.18-0.37$ & $<0.0001$ \\
\hline Hispanic & 0.28 & 0.07 & 1.32 & $1.15-1.52$ & 0.0002 \\
\hline \multicolumn{6}{|l|}{ Individual income, US\$ } \\
\hline 0-19000 & 0.00 & 0.00 & 1.00 & $1.00-1.00$ & - \\
\hline $20000-34000$ & -0.04 & 0.06 & 0.96 & $0.85-1.07$ & 0.441 \\
\hline $35000-69000$ & 0.18 & 0.06 & 0.84 & $0.75-0.94$ & 0.0025 \\
\hline$>70000$ & -0.49 & 0.10 & 0.61 & $0.51-0.75$ & $<0.0001$ \\
\hline
\end{tabular}

impulsive symptoms, considered separately, were. However, these results reflect a different pattern in men and women: whereas the number of hyperactive, impulsive or inattentive symptoms before 18 years was significantly associated with current obesity in women, none of the three symptom dimensions was significantly associated with obesity in men, although the association with the number of impulsive symptoms approached significance $(P=0.061)$. Given the current debate on how best to operationalise ADHD diagnostic criteria for adults, ${ }^{29}$ our results highlight the value of considering a dimensional approach.

Although the retrospective report of ADHD symptoms cannot strongly support causality, our results are consistent with the hypothesised role of both impulsivity and inattention in increasing the risk of obesity, at least in females. As for impulsivity, it has been proposed that both deficient inhibitory control and delay aversion reinforce abnormal eating behaviours, which, in turn, would increase the likelihood of obesity. ${ }^{30}$ Inattention and associated poor planning capacities might cause difficulties in adhering to regular eating patterns, favouring abnormal eating behaviours. ${ }^{30}$ Individuals with ADHD may also be relatively inattentive to internal signs of hunger and satiety. ${ }^{4}$ Inattention and associated deficient planning capacities may also interfere with dietary regimes in individuals with obesity who wish to decrease their body weight. ${ }^{4}$

Contrary to our expectation, we also found that the number of hyperactive symptoms before age 18 was significantly associated 
with obesity in women, even after controlling for possible confounders. This association could seem counterintuitive if one assumes that motor hyperactivity increases energy expenditure, thus favouring weight loss. However, it is well known that the motor hyperactivity of ADHD is not constant but is modulated by the context. Of note, little difference in hyperactivity levels between children with ADHD and healthy comparisons was detected while watching television ${ }^{31}$ and children with ADHD watch more television and engage in less physical activity than comparisons. ${ }^{12}$ Further studies are needed to better understand if and how the hyperactive dimension of ADHD can contribute to weight gain in women.

The association between ADHD symptoms before age 18 and current obesity in women but not in men is consistent with a previous study by van Egmond-Frohlich et al in adolescents where separate analyses by gender were performed. ${ }^{10}$ As van EgmondFrohlich and coworkers suggest, this gender difference may be accounted for by several factors, including the following.

(a) The prevalence of dysregulated eating disorders and, in particular, of binge eating disorder is higher in girls than in boys. $^{32}$

(b) Girls are under greater cultural pressure to be thin and thus to diet than boys. ${ }^{32}$ Since ADHD has been shown to be a significant barrier to weight loss, ${ }^{7}$ girls with high levels of ADHD symptoms are more prone to fail during their efforts to lose weight.

(c) Restrained eating is more frequent in girls compared with boys. ${ }^{32}$ High levels of impulsivity are associated with rebound effect after restrained eating, and there is evidence showing that in girls, but not in boys, higher preoccupation with weight predicted the development of overweight/ obesity. ${ }^{33}$

Therefore, impulsivity may lead to excessive overeating after food restriction, favouring weight gain in girls.

Our results have relevant clinical and public health implications, providing a strong rationale for longitudinal studies assessing the value of screening and treatment interventions aimed at preventing obesity by addressing impulsivity, inattention and hyperactivity in girls, regardless of the presence of a categorical diagnosis of $\mathrm{ADHD}$. Inattention and related impaired executive functions, as well as impulsivity that hamper the appropriate adherence to a regular diet regime, might be mistakenly attributed to laziness and 'character problems', adding to the stigmatisation of individuals with obesity. ${ }^{4}$ Therefore, treatments focused on these symptoms are paramount to decrease stigma associated with obesity. The pharmacological treatment of comorbid ADHD with psychostimulants leads to significant long-term weight loss in individuals with a lengthy history of weight loss failure, because of the positive effects of treatment on self-directedness, persistence and noveltyseeking behaviours rather than the temporary anorexigenic effects of psychostimulants. ${ }^{7}$ As a complement to pharmacological treatment, cognitive-behavioural therapy (CBT) has also been reported to be effective for ADHD in adults. ${ }^{34}$ Our study provides a rationale to assess the efficacy of pharmacological treatment as well as of CBT addressing ADHD symptoms in girls, regardless of the presence of a categorical diagnosis, in order to prevent weight gain in adulthood.

From a research standpoint, our findings suggest the following.

(a) A dimensional approach focused on ADHD symptom dimensions, rather than on ADHD as a category, might be a fruitful avenue in future research on the psychopathological and neurobiological mechanisms underlying the link between ADHD and obesity. This is in line with the Research
Domain Criteria initiative of the National Institute of Mental Health, ${ }^{35}$ which aims to establish the genetic and neural correlates of dimensions of observable behaviours, extending and complementing current categorical diagnoses.

(b) It is pivotal to consider gender differences when assessing the relationship between ADHD symptoms and risk for obesity.

\section{Limitations}

Our findings should be considered in the light of study limitations. The assessment of childhood ADHD, as well as of remission/ persistence of ADHD symptoms, was retrospective. However, the reliability and validity of retrospective report of ADHD symptoms have been shown in several studies (for example Epstein \& Kollins, ${ }^{36}$ Ward et $a l^{37}$ ), although others have shown that retrospective report can lead to underestimation ${ }^{38}$ or overestimation of ADHD prevalence. ${ }^{39}$ Attention-deficit hyperactivity disorder symptoms and related impairments were endorsed directly by the participants, without additional information from a third person or school reports, although the appropriateness of the use of external reports for the diagnosis of ADHD in adults is still debated in the field. ${ }^{29}$ This may help explain the lower prevalence of ADHD found in this study compared with the rate (approximately 4\%) reported when additional information from other individuals is sought, as discussed in our previous study. ${ }^{22}$ The items of the AUDADIS-IV interview operationalised the 18 items of the DSM-IV criterion A, which were originally developed to diagnose ADHD in school-aged children and may not be sensitive enough to diagnose ADHD in adults. This may have led to an underestimation of the ADHD prevalence rate. The availability, in the near future, of the upcoming ADHD DSM-5 criteria, providing examples of ADHD symptoms applicable across the lifespan, will help address this issue.

Weight and height were self-reported. It is unlikely and we are not aware of any empirical evidence that self-report biases reports of weight and height by individuals with and without ADHD differentially. Moreover, there is evidence that in the US population $<60$ years, self-reported weight and height are an accurate estimation of directly measured values. ${ }^{40}$

Another limitation is that the NESARC did not include a systematic assessment of eating disorders, which may have provided useful insight into the pathways linking ADHD and obesity, as well as of sleep disturbances, which may be associated with $\mathrm{ADHD}^{41}$ and have been shown to contribute to weight gain. ${ }^{42}$ Finally, we could not control for the possible effect of psychotropic drugs on weight status. However, given the anorexigenic effects of psychostimulant treatment, ${ }^{42}$ the most common ADHD treatment, their effects would not be expected to lead to a spurious association between ADHD and obesity.

\section{Implications and future perspectives}

Our results may have important implications for clinical practice, public health policy and future research. Although ADHD in adulthood was associated with obesity, the association did not hold after controlling for sociodemographic factors and psychiatric comorbidities. This suggests that programmes aimed at reducing obesity in adults with ADHD should target comorbid disorders. On the other hand, the association of ADHD symptoms before age 18 with obesity rates in women, even after controlling for sociodemographic characteristics and comorbid mental disorders, should prompt clinicians to systematically monitor for obesity risk in girls with high levels of ADHD symptoms, regardless of the presence of a formal diagnosis of ADHD and other comorbid psychiatric disorders. Longitudinal studies should 
be conducted to assess the efficacy of the treatment of ADHD symptoms in girls in order to prevent weight gain in adulthood.

Future research adopting a dimensional approach to ADHD, complementing a categorical one, could advance our knowledge of the relationship between ADHD symptoms and obesity. Given the clinical relevance and the impact of both ADHD and obesity in terms of public health, further research on their association and their management when they co-occur should be encouraged.

Samuele Cortese, MD, PhD, Phyllis Green and Randolph Cowen Institute for Pediatric Neuroscience, Child Study Center of the NYU Langone Medical Center, New York, USA, Child Neuropsychiatry Unit, G B Rossi Hospital, Department of Life and Reproduction Sciences, Verona University, Verona, Italy, and UMR_S INSERM U 930, ERL 3106, François-Rabelais University, Child Psychiatry Centre, University Hospital, Tours, France; Stephen v. Faraone, PhD, Department of Psychiatry and of Neuroscience and Physiology, State University of New York Upstate Medical University, Syracuse, New York, USA; Silvia Bernardi, MD, shuai Wang, PhD, Carlos Blanco, MD, PhD, Department of Psychiatry, Columbia University/New York State Psychiatric Institute, New York, USA

Correspondence: Samuele Cortese, MD, PhD, Child Neuropsychiatry Unit, Department of Life and Reproduction Sciences, Verona University, Verona, Italy. Email: samuele.cortese@gmail.com

First received 3 Nov 2012, final revision 19 Mar 2013, accepted 27 Mar 2013

\section{Funding}

The National Epidemiologic Survey on Alcohol and Related Conditions was sponsored by the National Institute on Alcohol Abuse and Alcoholism with supplemental support from the National Institute on Drug Abuse. Work on this manuscript was supported by 'Marie Curie' grant PIOF-253103 from the European Commission (S.C.), NIH grants DA019606 and DA023200 (C.B.), and New York State Psychiatric Institute and $\mathrm{NIH}$ gran R25MH086466 (S.B.)

\section{References}

1 Polanczyk G, de Lima MS, Horta BL, Biederman J, Rohde LA. The worldwide prevalence of ADHD: a systematic review and metaregression analysis. Am J Psychiatry 2007; 164: 942-8.

2 Faraone SV, Biederman J, Mick E. The age-dependent decline of attention deficit hyperactivity disorder: a meta-analysis of follow-up studies. Psychol Med 2006; 36: 159-65.

3 Biederman J, Faraone SV. Attention-deficit hyperactivity disorder. Lancet 2005; 366: 237-48

4 Cortese S, Vincenzi B. Obesity and ADHD: clinical and neurobiological implications. Curr Top Behav Neurosci 2012; 9: 199-218.

5 Clinical Guidelines on the Identification, Evaluation, and Treatment of Overweight and Obesity in Adults-The Evidence Report. National Institutes of Health. Obes Res 1998; 6 (suppl 2): 51S-209.

6 Wang YC, McPherson K, Marsh T, Gortmaker SL, Brown M. Health and economic burden of the projected obesity trends in the USA and the UK. Lancet 2011; 378: 815-25.

7 Levy LD, Fleming JP, Klar D. Treatment of refractory obesity in severely obese adults following management of newly diagnosed attention deficit hyperactivity disorder. Int J Obes (Lond) 2009; 33: 326-34.

8 Simon GE, Von KM, Saunders K, Miglioretti DL, Crane PK, van Belle G, et al. Association between obesity and psychiatric disorders in the US adult population. Arch Gen Psychiatry 2006; 63: 824-30.

9 Fuemmeler BF, Ostbye T, Yang C, McClernon FJ, Kollins SH. Association between attention-deficit/hyperactivity disorder symptoms and obesity and hypertension in early adulthood: a population-based study. Int J Obes (Lond) 2011; 35: 852-62.

10 van Egmond-Frohlich AW, Widhalm K, de Zwaan M. Association of symptoms of attention-deficit/hyperactivity disorder with childhood overweight adjusted for confounding parental variables. Int J Obes (Lond) 2012; 36: 963-8.

11 Byrd HC, Curtin C, Anderson SE. Attention-deficit/hyperactivity disorder and obesity in US males and females, age 8-15 years: National Health and Nutrition Examination Survey 2001-2004. Pediatr Obes 2013, January 16 (epub ahead of print).

12 Kim J, Mutyala B, Agiovlasitis S, Fernhall B. Health behaviors and obesity among US children with attention deficit hyperactivity disorder by gender and medication use. Prev Med 2011; 52: 218-22.

13 Grant BF, Stinson FS, Dawson DA, Chou SP, Ruan WJ, Pickering RP. Co-occurrence of 12-month alcohol and drug use disorders and personality disorders in the United States: results from the National Epidemiologic Survey on Alcohol and Related Conditions. Arch Gen Psychiatry 2004; 61: 361-8.

14 Grant BF, Stinson FS, Dawson DA, Chou SP, Dufour MC, Compton W, et al. Prevalence and co-occurrence of substance use disorders and independent mood and anxiety disorders: results from the National Epidemiologic Survey on Alcohol and Related Conditions. Arch Gen Psychiatry 2004; 61: 807-16.

15 Grant BF, Goldstein RB, Chou SP, Huang B, Stinson FS, Dawson DA, et al. Sociodemographic and psychopathologic predictors of first incidence of DSM-IV substance use, mood and anxiety disorders: results from the Wave 2 National Epidemiologic Survey on Alcohol and Related Conditions. $\mathrm{Mol}$ Psychiatry 2009; 14: 1051-66.

16 Grant B, Dawson D, Hasin D. The Wave 2 National Epidemiologic Survey on Alcohol and Related Conditions Alcohol Use Disorder and Associated Disabilities Interview Schedule-DSM-IV Version. National Institutes of Health, 2001 (http://www.niaaa.nih.gov.).

17 American Psychiatric Association. Diagnostic and Statistical Manual of Mental Disorders (4th edn) (DSM-IV). APA, 1994.

18 Kieling C, Kieling RR, Rohde LA, Frick PJ, Moffitt T, Nigg JT, et al. The age at onset of attention deficit hyperactivity disorder. Am J Psychiatry 2010; 167: $14-6$.

19 American Psychiatric Association. Diagnostic and Statistical Manual of Mental Disorders (4th edn, text revision) (DSM-IV-TR). APA, 2000.

20 Ruan WJ, Goldstein RB, Chou SP, Smith SM, Saha TD, Pickering RP, et al. The alcohol use disorder and associated disabilities interview schedule-IV (AUDADIS-IV): reliability of new psychiatric diagnostic modules and risk factors in a general population sample. Drug Alcohol Depend 2008; 92 : 27-36.

21 World Health Organization. Obesity and Overweight. Factsheet 311. WHO, 2013 (http://www.who.int/mediacentre/factsheets/fs311/en/).

22 Bernardi S, Faraone SV, Cortese S, Kerridge BT, Pallanti S, Wang S, et al. The lifetime impact of attention deficit hyperactivity disorder: results from the National Epidemiologic Survey on Alcohol and Related Conditions (NESARC). Psychol Med 2012; 42: 875-87.

23 Rojo L, Ruiz E, Dominguez JA, Calaf M, Livianos L. Comorbidity between obesity and attention deficit/hyperactivity disorder: population study with 13-15-year-olds. Int J Eat Disord 2006; 39: 519-22.

24 Pagoto SL, Curtin C, Lemon SC, Bandini LG, Schneider KL, Bodenlos JS, et al. Association between adult attention deficit/hyperactivity disorder and obesity in the US population. Obesity (Silver Spring) 2009; 17: 539-44.

25 Rosler M, Retz W, Thome J, Schneider M, Stieglitz RD, Falkai P. Psychopathological rating scales for diagnostic use in adults with attentiondeficit/hyperactivity disorder (ADHD). Eur Arch Psychiatry Clin Neurosci 2006; 256 (suppl 1): i3-11.

26 Barkley RA, Murphy KR, Fischer M. ADHD in Adults: What the Science Says. Guilford, 2007.

27 Centers for Disease Control and Prevention. Vital signs: state-specific obesity prevalence among adults - United States, 2009. MMWR Morb Mortal Wkly Rep 2010; 59: 951-5.

28 de Zwaan M, Gruss B, Muller A, Philipsen A, Graap H, Martin A, et al. Association between obesity and adult attention-deficit/hyperactivity disorder in a German community-based sample. Obes Facts 2011; 4 204-11.

29 Matte B, Rohde LA, Grevet EH. ADHD in adults: a concept in evolution Atten Defic Hyperact Disord 2012; 4: 53-62.

30 Davis C, Levitan RD, Smith M, Tweed S, Curtis C. Associations among overeating, overweight, and attention deficit/hyperactivity disorder: a structural equation modelling approach. Eat Behav 2006; 7: 266-74.

31 Porrino L, Rapoport JL, Behar D, Sceery W, Ismond DR, Bunney Jr WE. A naturalistic assessment of the motor activity of hyperactive boys. I Comparison with normal controls. Arch Gen Psychiatry 1983; 40: 681-7.

32 Neumark-Sztainer D, Wall M, Larson NI, Eisenberg ME, Loth K. Dieting and disordered eating behaviors from adolescence to young adulthood: findings from a 10-year longitudinal study. J Am Diet AssoC 2011; 111: 1004-11.

33 Haines J, Kleinman KP, Rifas-Shiman SL, Field AE, Austin SB. Examination of shared risk and protective factors for overweight and disordered eating among adolescents. Arch Pediatr Adolesc Med 2010; 164: 336-43.

34 Safren SA, Sprich S, Mimiaga MJ, Surman C, Knouse L, Groves M, et al. Cognitive behavioral therapy vs relaxation with educational support for medication-treated adults with ADHD and persistent symptoms: a randomized controlled trial. JAMA 2010; 304: 875-80.

35 Insel T, Cuthbert B, Garvey M, Heinssen R, Pine DS, Quinn K, et al. Research domain criteria (RDoC): toward a new classification framework for research on mental disorders. Am J Psychiatry 2010; 167: 748-51. 
36 Epstein JN, Kollins SH. Psychometric properties of an adult ADHD diagnostic interview. J Atten Disord 2006; 9: 504-14.

37 Ward MF, Wender PH, Reimherr FW. The Wender Utah Rating Scale: an aid in the retrospective diagnosis of childhood attention deficit hyperactivity disorder. Am J Psychiatry 1993; 150: 885-90.

38 Barkley RA, Fischer M, Smallish L, Fletcher K. The persistence of attention-deficit/hyperactivity disorder into young adulthood as a function of reporting source and definition of disorder. J Abnorm Psychol 2002; 111: $279-89$.

39 Mannuzza S, Klein RG, Klein DF, Bessler A, Shrout P. Accuracy of adult recall of childhood attention deficit hyperactivity disorder. Am J Psychiatry 2002; 159: $1882-8$.
40 Kuczmarski MF, Kuczmarski RJ, Najjar M. Effects of age on validity of self-reported height, weight, and body mass index: findings from the Third National Health and Nutrition Examination Survey, 1988-1994. J Am Diet Assoc 2001; 101: 28-34.

41 Bijlenga D, van der Heijden KB, Breuk M, van Someren EJW, Lie MEH, Boonstra AM, et al. Associations between sleep characteristics, seasonal depressive symptoms, lifestyle, and ADHD symptoms in adults. J Atten Disord 2013; 17: 261-75.

42 Taheri S, Lin L, Austin D, Young T, Mignot E. Short sleep duration is associated with reduced leptin, elevated ghrelin, and increased body mass index. PLoS Med 2004; 1: e62.

\section{psychiatry} in literature

\section{Reflections on Beckett's Murphy}

\section{Larkin Feeney, Peter McCarron}

Although better known for his existentialist drama in which the nature of sanity is a recurrent theme, it is in his fiction that Samuel Beckett most directly addresses psychiatry. Unafraid to use for his raw materials life's grimmest situations - loneliness, despair, the decay of both body and mind - Beckett fashions them into prose masterpieces. As in real life they are often relatively plotless and despite their harrowing themes, darkly and philosophically comic.

In his 1938 second novel, Murphy, the eponymous hero (and also the fictional father of Ken Kesey's more animated Randall P. MacMurphy) shuns almost every aspect of conventional existence - work, respectability, marriage and money. Nevertheless, to quell the anxious urgings of his lover, Celia Kelly, Murphy takes up employment as a male nurse at the Magdalen Mental Mercyseat - clearly modelled on the Bethlem Royal Hospital - in north London. This role might appear implausible; Murphy after all is chronically depressed, socially phobic, alogic, avolitional, devoid of appetite and reduced to spending much of his time in darkness, bound naked to a rocking chair. However, he demonstrates surprising aptitude for the job: 'Another melancholic, convinced that his intestines had turned to twine and blotting-paper, would only eat when Murphy held the spoon'. Beckett questions what it means to be sane, depicting the underqualified and sadistic nurses as the real lunatics and the psychiatrists as having 'text-book attitude(s)' and 'complacent scientific conceptualism'; only the 'seedy solipsist' Murphy can meaningfully connect with the patients. Although the institution offers Murphy temporary sanctuary from the commotion of the outside world, his pursuers close in and, driven to greater despair after a circular game of chess with Mr Endon ('the most biddable little gaga in the entire institution'), Murphy wanders away from his post and later self-immolates in his hospital garret: 'The gas went on in the w.c., excellent gas, superfine chaos. Soon his body was quiet'.

Beckett visited his friend Geoffrey Thompson at Bethlem Royal Hospital in Kent while researching the novel and he demonstrates considerable insight into the operations of a psychiatric hospital. Irish psychiatrists may also nod as they note that Beckett knew the correct respective roles of two well-known South Dublin mental institutions in Stillorgan and Dundrum. In addition, Beckett had recently begun psychoanalysis with Wilfred Bion and the novel can be read as a metaphor for the process of his treatment. Similar characters to Murphy appear in many of Beckett's later works, always in retreat from so-called normal life, and struggling pitifully and often grotesquely to uncover meaning, where, for Beckett, no meaning existed. Murphy is a highly entertaining and accessible introduction to the themes that continued to preoccupy Beckett in his later, increasingly spare and experimental prose works, most notably the trilogy of Molloy, Malone Dies and The Unnamable. 